\title{
Construcción y reconstrucción del objeto de estudio en la investigación educativa
}

Construction and Reconstruction of the Object of Study in Educational Research

\section{Volumen 19, Número 3 \\ Setiembre-Diciembre \\ pp. 1-21}

Gabriel Alejandro Álvarez Hernández

Citar este documento según modelo APA

Álvarez Hernández, Gabriel Alejandro. (2019). Construcción y reconstrucción del objeto de estudio en la investigación educativa. Revista Actualidades Investigativas en Educación, 19(3), 1-21. Doi. 10.15517/aie.v19i3.38795 


\title{
Construcción y reconstrucción del objeto de estudio en la investigación educativa \\ Construction and Reconstruction of the Object of Study in Educational Research
}

\section{Gabriel Alejandro Álvarez Hernández ${ }^{1}$}

\begin{abstract}
Resumen: El presente ensayo plantea la discusión y el debate en torno a la construcción y reconstrucción del objeto de estudio en investigaciones en ciencias sociales y humanas, más específicamente en el campo educativo. Primero, a manera de preludio, se bosquejan una serie de reflexiones sobre las implicaciones subjetivas, sociales y culturales como bases de la concepción de una realidad. Posteriormente, se esbozan premisas y debates en relación con la ciencia moderna del siglo XX hasta nuestros tiempos, una revisión que recupera, entre otras cosas, la evolución del pensamiento, el racionalismo, la lógica y el diseño de ciencias desde las tradiciones Galileana y Aristotélica y su influencia en los procesos de construcción del conocimiento. Luego, se destaca la importancia de la ontología como principio para dar respuesta a la realidad, y a la epistemología como inicio de configuraciones conceptuales que fundamentan el acercamiento al objeto de estudio. Por último, se cierra destacando los aportes de teóricos que proponen maneras de abordar la realidad y construir el objeto, asimismo, se mencionan algunos planteamientos epistemológicos emergentes que abonan la discusión.
\end{abstract}

Palabras clave: ontología, epistemología, investigación educativa, objeto de estudio.

\begin{abstract}
This essay raises the discussion and debate around the construction and reconstruction of the object of study in social and human sciences research, more specific in the educational field. First, as a prelude, a series of reflections on subjective, social and cultural implications as the basis for the conception of a reality are outlined. Subsequently, premises and debates are outlined in relation to modern science of the twentieth century to our times, a review that recovers, among other things, the evolution of thought, rationalism, logic and science design from the Galilean and Aristotelian traditions and its influence on the processes of knowledge construction. Then, it emphasizes the importance of ontology as a principle to respond to reality, and to epistemology as the beginning of conceptual configurations that support the approach to the object of study. Finally, it closes highlighting the contributions of theorists who propose ways of approaching reality and building the object, they say, some emerging epistemological approaches that support the discussion are mentioned.
\end{abstract}

Keywords: ontology, epistemology, educational research, object of study.

\author{
${ }^{1}$ Profesor de la Universidad Pedagógica Nacional, México. \\ Dirección electrónica: gabo.alvarezh@gmail.com \\ Ensayo recibido: 30 de marzo, 2019 \\ Enviado a corrección: 13 de junio, 2019 \\ Aprobado: 19 de agosto, 2019
}




\section{Introducción}

En la primera parte se abre con un preludio que expone reflexiones sobre las implicaciones subjetivas, sociales y culturales de la realidad. De modo que estas son elementos que están presentes en el modo en que las personas se relacionan con los fenómenos sociales y cómo es que las personas que hacen investigación se ven influenciadas al momento de construir su potencial objeto de estudio.

En la segunda parte titulada Ciencia: Debates y premisas para la construcción del objeto de estudio, se realiza un recuento del devenir de la ciencia moderna y los elementos que le dan cuerpo, se consideran los conceptos de ciencias Galileana y Aristotélica como punto de partida de la reflexión y constitución de espacios epistémicos que dan pauta a consideraciones de orden ontológico, epistemológico y metodológico.

La tercera parte, llamada Realidad y construcción del objeto de estudio: Ontología y epistemología, destaca tres premisas de la investigación científica: ontología, epistemología y metodología; no obstante, se desarrollan solo las primeras dos, pues corresponden a la realidad y posibilidades de construir conocimiento respectivamente. Estas son abordadas en el marco de los tres paradigmas más concurridos: el positivista, el interpretativo y el sociocrítico.

Las Reflexiones es el apartado que cierra este manuscrito, en él se presentan aportes que son propositivos con los principios y las formas a partir de las cuales se puede construir un objeto de estudio. Luego se mencionan algunas tendencias alternativas, donde la epistemología anarquista de Paul Feyerabend, la semiótica de Humberto Eco y la epistemología decolonizadora de Boaventura De Sousa, como posibilidades epistemológicas y ontológicas que permiten otra visión a las mencionadas y descritas a lo largo del texto. Por último, se medita sobre la educación como un espacio en el que la experiencia vivida es un elemento sustantivo que interviene en los procesos de construcción y reconstrucción del objeto de estudio, pues los espacios escolares se fundan como sitios de múltiples interpretaciones, de modo que quien hace investigación tiene que asumir que la actividad investigativa es también una acción profundamente humana. 


\section{Preludio}

"Mi relato será fiel a la realidad o, en todo caso, a mi recuerdo personal de la realidad, lo cual es lo mismo." Jorge Luis Borges

Una de las características de la investigación, que puede ser de mayor controversia para quienes intentan realizar esta actividad llamada científica, es, precisamente, la Construcción y reconstrucción del objeto de estudio. Comprenderlo y explicarlo pone en juego toda una serie de elementos epistemológicos y ontológicos que subyacen o pretenden subyacer en la manera en que un investigador se comporta con su objeto de estudio. Más aún, se juegan las capacidades de una persona que se enfrenta a algo y al mismo tiempo a sí mismo, con la intención de investigarlo y dar cuentas a terceros.

El campo educativo, como espacio de subjetividades, de encuentros y desencuentros, consensos y disensos, se destaca por su complejidad y multiplicidad de fenómenos, los cuales requieren y exigen ser investigados, de modo que el problematizar y construir el objeto de estudio se vuelve una tarea especialmente complicada, pues el contexto da pautas que dan singularidad. Construir el objeto de estudio en los sitios escolares confiere a quien hace investigación la sinuosa tarea de recuperar la diversidad y articular cada elemento en lo que se entenderá como objeto de estudio.

Investigar los fenómenos educativos no es solo una actividad que se desarrolla como la puesta en práctica de una técnica, la aplicación correcta de instrumentos o el seguir cauta y disciplinadamente una serie de pasos; en ocasiones, tampoco es un acto metódico y estricto, a veces ni siquiera se puede contemplar la posibilidad de que haya un manual que respetar devotamente. A veces, el acto de investigar es tan solo pararse frente a una realidad, ante un fenómeno y contemplarlo, aprehenderlo, vivirlo, hacerlo experiencia propia; a veces, es ser tan solo pasivos, pero no pasivos en el sentido de no tener actividad, sino en el de permitirse que las cosas le pasen a uno (Larrosa, 2003), una pasividad que intenta desprendernos, aunque sea por un instante, de ese impulso a veces ciego y tempestuoso de racionalizar todo lo que acontece. ${ }^{2}$

\footnotetext{
${ }^{2}$ Aquí se precisa que se hace referencia a una investigación interpretativa, diferenciada de una empírico-analítica, o como suele también decirse en los manuales de investigación, lo cualitativo en oposición de lo cuantitativo (N. del A.)
} 
La frase que inicia este escrito expresa una especie de contradicción y afirmación al mismo tiempo, pues una persona relata un aspecto de la realidad que se intenta manifestar como verdad: lo que se cuenta, al menos para quien lo cuenta, es una verdad o eso pretende ser, pues es su recuerdo y de nadie más. Un investigador puede pararse frente a un fenómeno de la realidad, como lo es la educación, y encontrar ciertas explicaciones y describir lo que ahí pasa de un modo que para un interlocutor esa es una verdad, pero luego otro investigador puede pararse frente al mismo fenómeno y explicarlo y describirlo de un modo distinto, esto no quiere decir que uno esté diciendo mentiras y el otro la verdad, es que cada uno ve algo diferente porque su historia de vida, deseos, intenciones, sus experiencias, saberes y conocimientos entran en juego, el recuerdo en dado caso es diferente. En La Nausea de Jean Paul Sartre (2012) el protagonista Antoine Roquentin comienza inmediatamente por hacer una sugerencia:

Lo mejor sería escribir los acontecimientos cotidianamente. Llevar un diario para comprenderlos. No dejar escapar los matices, los hechos menudos, aunque parezcan fruslerías, y sobre todo clasificarlos. Es preciso decir cómo veo esta mesa, la calle, la gente, mi paquete de tabaco, ya que es esto lo que ha cambiado. Es preciso determinar exactamente el alcance y la naturaleza de este cambio. (2012, p. 7)

Al decir "la naturaleza del cambio" se abre de manera implícita una clave en esta oración para entender por qué la sugerencia: la realidad humana y social es por naturaleza cambio, transformación, vicisitud, metamorfosis y todos los sinónimos que podamos encontrar y esto emana una especie de sin sentido, y al parecer para Antoine Roquentin es preciso llevar registro de los acontecimientos, de modo que no escape nada, ni los detalles más mínimos, para poder entender por qué las cosas cambian o si es que cambiaron, o bien, entender que lo que cambió fue la manera de percibir el mundo, pues en algún momento él duda de su percepción: "Lo curioso es que no estoy nada dispuesto a creerme loco; hasta veo con evidencia que no lo estoy: todos los cambios conciernen a los objetos. Por lo menos quisiera estar seguro de esto" (Sartre, 2012, p. 8).

Independientemente de los análisis filosóficos que hay de esta obra literaria, se puede reflexionar que en el mundo de lo social y lo humano las percepciones se expresan en gran medida, el aparato subjetivo se despliega hacia el objeto a investigar y ese despliegue lleva en su interior experiencias, deseos, intenciones, expectativas, creencias, intereses, etcétera, 
incluso en ocasiones un investigador duda de su objeto de estudio; en esta versión de lo subjetivo va la argumentación de una persona que intenta hacer de un fragmento de la realidad el centro de su actividad investigativa. De esta reflexión, se puede decir que los espacios escolares, donde se elabora la experiencia educativa protagonizada por la planta docente y estudiantes principalmente, son lugares donde las versiones de lo visto cambia irremediablemente según cada quien, las afirmaciones sobre el presunto objeto de estudio son siempre distintas.

En un cambio de orden de ideas, de lo ambiguo que simboliza lo subjetivo a lo sinuoso que constituye lo social, se puede deducir que la cultura es algo que también cambia constantemente; por ejemplo, las últimas 40,000 generaciones la humanidad fuimos una entidad nómada, deambulando por el mundo detrás del alimento y el agua, apenas con algunas herramientas que fueron las armas que permitieron la cacería y el fuego actuó como aliado.

Luego todo cambió cuando comenzó a tenerse registro de lo que se hacía y cómo se vivía, las pinturas rupestres de las Cuevas de Chauvet son testimonio de ello. Posteriormente algunas culturas, incluyendo las prehispánicas, pensaron que mirar al cielo y llevar un apunte detallado del movimiento de las estrellas fuera algo interesante, eso provocó que se lograra entender cuándo pasarían cada una de las cuatro estaciones del año y saber las migraciones de las manadas de animales y poder cazar con mayor eficiencia, además los equinoccios y solsticios fueron para algunas culturas eventos de celebración; consecuentemente se aprendió a domesticar a los animales y las plantas, así el alimento ya no se perseguía, sino que se producía. Concluyó una era nómada por una sedentaria, con la agricultura como socia se dio inicio a la civilización.

Surgió la escritura y jugó un papel importante, pues a partir del registro del pensamiento fue que se comenzó a trascender en la historia, ahora leemos textos con antigüedad de 2200 años A. de C. como la Epopeya de Gilgamesh hasta el último novel de literatura, un cambio revolucionario se dio con la escritura. El descubrimiento y conquista de América fue otro evento que marcó un antes y un después, más recientemente, eventos como los movimientos independistas y las revoluciones transforman el orden social y la cultura; las guerras, los consensos, disensos, los personajes históricos, unos vanagloriados y otros odiados, como sea, el cambio y la transformación de la cultura tiene muchas explicaciones, pero lo cierto y para fines de este trabajo escrito es que la cultura se 
constituye por un cambio constante e inevitable y los ejemplos para demostrarlo son numerosos.

La educación como un fenómeno social y cultural no escapa de esta dinámica, quien investiga, entre otras cosas, debe entender y aceptar que hay vicisitudes dialécticas, que hay acciones con implicaciones morales. Al comprender y reflexionar desde este planteamiento a la educación, sería un tanto absurdo pensar que los objetos de estudio son algo acabado, que están allí esperando ser estudiados como algo inamovible y estático, es caer en un reduccionismo y en la cosificación.

Ya sea por razones subjetivas o sociales, el acto educativo y los distintos fenómenos que convergen en este son aspectos que requieren una constante revisión y reelaboración, pero no en el distanciamiento de la mirada fría y objetiva, sino en la vivencia misma, en la posibilidad de hacer experiencia lo que otros viven, aunque sea por un instante, hecho que permite reconstruir lo que en algún momento se construyó: el objeto de estudio.

Alfred Schutz (2015) propuso una reflexión de orden fenomenológico social, este autor abre las puertas a una discusión que invita a repensar el modo en el que las personas versan sobre los fenómenos sociales a partir de dos grandes cuestionamientos: el primero surge en el momento en el que se establece una presunta neutralidad ante los sucesos sociales, el hecho de garantizar una simple descripción de la realidad sin que el investigador se vea involucrado, lo cual resulta ilusorio "Nada garantiza que el mundo que el actor presupone subjetivamente sea también incuestionable para el observador" (p. 107); el segundo, "[...] se refiere a la índole de la idealización y generalización que efectúa el especialista en ciencias sociales al describir las acciones que tienen lugar dentro del mundo social." (p. 108). De modo que la actuación de quien investiga a partir de la creencia de que los hechos sociales son algo dado e incuestionable y con tendencias a generalizar el pensamiento de los actores no es posible. Un componente de intersubjetividad es evidente y constante en la vida cotidiana y el campo educativo, recuperarlo e incorporarlo a la labor de la construcción y reconstrucción del objeto de estudio ayuda a entender que este es derivado, entre otras cosas, de la cotidianidad y las interpretaciones.

Cerrando este preludio, no queda más que aceptar con ánimo y entusiasmo que los objetos de estudio en el campo educativo son construcciones y reconstrucciones constantes, ya sea por un impulso subjetivo o por las circunstancias sociales y culturales, o por ambas. Será el cimiento epistemológico, la congruencia teórica y metodológica e incluso la narrativa 
del investigador, los ingredientes que van a permitir a terceros aceptar lo que en un momento se anuncia y sostiene como objeto de estudio y sus constantes reelaboraciones.

\section{Ciencia: debates y premisas para la construcción del objeto de estudio}

Lo versado en el preludio y hasta este instante exigen una aclaración o delimitación epistemológica, la cual se deduce incluso desde el título: Construcción y reconstrucción del objeto de estudio, donde el concepto "reconstrucción" nos emplaza en un ámbito preciso de la investigación científica: el lugar de las ciencias sociales y humanas, espacio en el que se desarrollan las reflexiones y discusiones a continuación expuestas. Por principio habría que esclarecer la dimensión epistemológica a la que pertenecen las ciencias sociales y humanas y donde también se encuentra la educación, al menos aquella versión que pudiera aceptar e incluso exigir una construcción y reconstrucción del objeto de estudio, es la interpretativa.

Los objetos de investigación científica, por principio, se asumen como referentes de estudio que superan en su elaboración más acabada las suposiciones de sentido común, en cambio se erigen como construcciones que sin duda engloban interpretaciones, pero entretejen conocimiento que se tiene sobre la realidad, esto es:

Los objetos de investigación científica no son empíricos, sino conceptuales; el investigador no "toma" problemas de la realidad, sino que los construye desde sus referentes interpretativos y el conocimiento previo de la realidad por investigar. Implica una actitud intelectual que supera la ilusión de que existen "objetos" preconstituidos, percibidos y valorados desde la intuición y el sentido común. (Torres y Jiménez, 2004, p.16)

El primer debate o discusión que hay que asumir es aquel que cuestiona el concepto mismo de ciencia, pero ¿dónde y cómo comienza el concepto de ciencia que es el que conocemos hoy en día? Se puede decir que sus albores están en la Modernidad, esta como impulso del Renacimiento y dada una paulatina caída de la iglesia como centro y eje de las meditaciones y comportamientos de la civilización en el mundo occidental desde la Edad Media, esto provocó un cambio en la cosmovisión de manera amplia: una nueva forma de pensar la vida, de relacionarse con el mundo se alza con el estandarte de la razón, el cogito cartesiano se vertebra como el principio de un racionalismo que sirvió de base para dar un giro de 180 grados a un esquema logocéntrico/racionalista. 
A partir de ese momento las luces de la razón son el faro que pretende iluminar y abrir el camino hacia el progreso y el orden, en contraposición de las reglas inquebrantables propias de la opresión y el totalitarismo de la iglesia fundamentalmente. La llustración, como movimiento filosófico, intelectual y cultural, promovió seriamente la reconfiguración de los proyectos sociales, políticos, económicos y científicos, propuso el conocimiento como la herramienta para combatir la ignorancia, la superstición, el absolutismo y la intolerancia. El siglo XVIII como el siglo de las luces fulguró por su fe y esperanza en el progreso, el razonamiento y la ciencia son la base para lograr la encomienda.

El tránsito hacia el siglo XX vino acompañado de una corriente ideológica configurada e impulsada por los aportes de Augusto Comte, Francis Bacon, David Hume, entre otros. El positivismo sería entonces el resultado de una guisa conceptual que incorpora al empirismo, el análisis, el deductivismo, la hipótesis y una fuerte terminología que afirmaría que el conocimiento del mundo sería consecuencia de un razonamiento y un quehacer cientificista, del estudio del mundo como un ente natural regido por leyes que era necesario encontrar y poner al servicio de la humanidad. El positivismo sería la epistemología de una ciencia empírico-analítica (o ciencia moderna) que llegó al siglo XX como la panacea, la fe de la humanidad estaba depositada ciegamente en esta, cuenta y predica un objetivismo:

[...] el objetivismo con respecto al conocimiento humano es una concepción que hace hincapié en que los datos del conocimiento, desde las proposiciones simples a las teorías complejas, tienen prioridades y características que trascienden las creencias y los estados de conciencia de los individuos que las conciben y contemplan. (Chalmers, 1992, p. 159)

El siglo XX comenzó con una inercia provocada por los procesos de industrialización, donde la reconfiguración de las relaciones sociales se dio en la lógica de la producción, distribución y consumo, del trabajo y la mano de obra. En ese contexto, la ciencia y la técnica, como señala Habermas (1984), se unen a la dinámica de la industrialización, momento en que las decisiones que antes eran políticas o prácticas se vuelven "técnicas", de modo que esa ciencia y tecnología se introducen en la vida cotidiana, en la cultura, como las antiguas ideologías, fueron el punto de acción sobre el mundo para su dominio en beneficio de la humanidad y la vida misma. La ciencia moderna encuentra los espacios de desarrollo y proliferación en las distintas instituciones humanas, donde, desde luego, está la educativa, 
así las escuelas en general y la Universidad en específico se traducen en espacios sí formativos, pero también de gremios científicos. La ciencia y aquellos que la predican se asumen con cierta reverencia y magnificación en el estatus social, así:

La llustración del siglo XVIII hizo a la gente más madura ante la Iglesia. Un instrumento esencial para conseguir esta madurez fue un mayor conocimiento del hombre y del mundo. Pero las instituciones que crearon y expandieron los conocimientos, muy pronto condujeron a una nueva especie de inmadurez. Hoy se acepta el veredicto de científicos o de otros expertos con la misma reverencia propia de débiles mentales que se reservaba antes a obispos y cardenales, y los filósofos, en lugar de criticar este proceso, intentan demostrar su «racionalidad» interna. (Feyerabend, 1996, p. 60)

En el mismo siglo $\mathrm{XX}$ se dieron una serie de discusiones epistemológicas que tuvieron como inicio la ruptura de las formas de acercamiento al mundo social y humano de la ciencia moderna eminentemente positivista, discusiones protagonizadas por el Circulo de Viena y la Escuela de Frankfurt, Karl Popper y Theodor Adorno, pues la lógica de la explicación causal parecía ser suficiente para los fenómenos de la naturaleza, pero lo social y humano escapan de esta racionalidad, la educación en este marco reflexivo, exige planteamientos paradigmáticos que permitan un entendimiento profundo que responda a su complejidad. Las discusiones tuvieron como principio cuestionar la denominada ciencia, más aún ¿qué es "ciencia"? La respuesta parecía predominar con una resonancia mediática de mayor alcance, pues se relacionaba con una tradición filosófica Galileana, un modelo explicativo (Erklaren) que luego tuvo como rival otro modelo teleológico y comprensivo (Verstehen), en este último se acentúa la peculiaridad del objeto socio-histórico, psicológico y las formas de aproximación (hermenéutica, fenomenología, dialéctica, lingüística...) (Mardones y Ursua, 1995).

Hasta aquí encontramos dos océanos epistemológicos que dan pie a sus correspondientes conceptos de ciencia desde dos tradiciones: de la empresa empíricoanalítica o positivista la ciencia Galileana, de una guisa interpretativa una ciencia Aristotélica (Véase Tabla 1); las derivaciones metodológicas de estas serán aquellas que en el lenguaje 
cotidiano se nombran como cuantitativas y cualitativas ${ }^{3}$, las cuales contemplan un concepto de realidad y determinarán la relación del investigador con esta, de modo que la construcción del objeto de estudio tendrá matices específicos. A continuación se exponen tan solo algunos de los conceptos que configuran cada concepto de ciencia:

Tabla 1: Conceptos clave de la ciencia Galileana y la Aristotélica

\begin{tabular}{|cc|cc|}
\hline $\begin{array}{c}\text { Ciencia Galielana } \\
\text { (Positivista, empírico-analítica) }\end{array}$ & \multicolumn{2}{|c|}{$\begin{array}{c}\text { Ciencia Aristotélica } \\
\text { (Interpretativa, histórico- } \\
\text { hermenéutica) }\end{array}$} \\
\hline$\circ$ & Positivismo & $\circ$ & Hermenéutica \\
$\circ$ & Pragmatismos & $\circ$ & Fenomenología \\
$\circ$ & Empirismo & $\circ$ & Experiencia vivida \\
$\circ$ & Variables & $\circ$ & Sentido/significado \\
$\circ$ & Explicación & $\circ$ & Comprensión \\
$\circ$ & Lógica deductiva & $\circ$ & Lógica inductiva \\
$\circ$ & Conocimiento & $\circ$ & Saberes \\
$\circ$ & Objetividad & $\circ$ & Subjetividad \\
\hline
\end{tabular}

Fuente: Elaboración propia a partir de Mardones y Ursúa, 1995

¿Cuál es el concepto de ciencia que debe ocuparse para estudiar la educación y los múltiples fenómenos que la configuran? Ambos conceptos pueden ser ocupados, sus respectivas metodologías pueden acercarnos a lo educativo, estudios de trayectorias escolares como los de Tinto (1987) en Estados Unidos, de Garay (2004, 2008) y González (2011) en México son ejemplo de trabajos cuantitativos, por su parte los estudios etnográficos son bastos y representativos de enfoques cualitativos.

El devenir de la ciencia en el siglo XX y aún latente en el XXI tiene sus albores epistemológicos en general en estas dos tradiciones, no obstante, no todo queda ahí, luego los paradigmas comenzaron a estructurarse al estilo Thomas Kuhn (2000) y comenzó una configuración que terminó en tres grandes paradigmas, los cuales se van a abordar en el siguiente apartado, así como las paulatinas implicaciones en la construcción del objeto de estudio orientado al campo educativo.

\footnotetext{
3 Aquí se precisa, de acuerdo con Ducoing, que las investigaciones "cuantitativas" son continuamente circunscritas a epistemologías empírico-analíticas, y por su parte las "cualitativas" son del dominio de epistemologías de corte interpretativo "Pareciera, así, que, en este marco, los "números" son propiedad de los positivistas, y las cualidades de los interpretativos, en tanto que los trabajos de corte interpretativo pueden también hacer uso de elementos cuantitativos y, viceversa, los fundados en epistemologías positivistas pueden movilizar dispositivos cualitativos." (2016, p. 15) En este trabajo no se intenta superponer cantidades o cualidades a los marcos metodológicos, sino establecer las configuraciones conceptuales de una y otra epistemología concentrando los esfuerzos en lo que sería el concepto de realidad y la posición que toma el investigador al respecto de cada cual.
} 


\section{Realidad y construcción del objeto de estudio: Ontología y epistemología}

Dos posturas se definen y consolidan en el siglo XX, dos conceptos de ciencia que incorporan bases conceptuales que servirán como bases epistemológicas, ontológicas y metodológicas para lo que después será el quehacer investigativo según corresponda. Los acercamientos a la realidad serán marcos de acción que propongan principios para la forma en que quien investiga se acerca a su objeto de estudio y lo construye. En este siglo, la investigación consolida su rigor, su metodología, sus principios e incluso la ideología desde la que se gesta. La ciencia será fe para el mundo occidental, la auténtica posibilidad de encontrar la verdad donde quiera que esté.

Para Domínguez (2007) existen múltiples acercamientos teóricos a la realidad, cada uno representado por autores y autoras que en su parcela de pensamiento abonan de un modo singular aspectos que han de considerarse como nodales en la manera de construir conocimiento, la misma autora destaca el aporte de Díaz Barriga (1997), quien contrapone teoría del conocimiento y teoría de la ciencia, donde la primera puede entenderse como una postura constructivista y la segunda es positivista; afirma que la teoría de la ciencia no puede dar cuentas de un objeto de conocimiento social específico, la razón estriba en que se apoya de la explicación, dejando de lado aspectos de corte comprensivo, en respuesta encuentra a la hermenéutica comprensiva como alternativa y con aristas más encontradas con una postura constructivista. Así, la construcción del objeto de estudio, sobre todo en el campo social y humano y al cual pertenece la educación, reposa epistemológicamente en una filosofía hermenéutica.

Cada una de estas configuraciones mantiene un concepto de realidad más o menos general, una ontología que propone una relación con el mundo y consecuentes implicaciones al campo de la investigación. No obstante, el siglo XX trajo consigo, en estas discusiones epistemológicas, la yuxtaposición de paradigmas que vendrían tanto a abonar la discusión como a precisar marcos de acción científica, paradigmas en el sentido de Thomas Kuhn (2000), quien los definió como los conceptos clave en los que se apoya una ciencia y que a su vez guían la investigación, tres son los paradigmas a ser los estelares en la investigación 
científica en el siglo XX y con presencia aún en el $X X I$ : el post-positivista, ${ }^{4}$ el interpretativo y el sociocrítico; el primero y el segundo, como se puede deducir, son consecuencia directa de las tradiciones Galileana y Aristotélica respectivamente, en cambio, el paradigma sociocrítico, en su estructura filosófica más profunda, incorpora la dialéctica hegeliana como base sustantiva con ambiciones de síntesis superadora.

Los tres paradigmas de investigación mencionados responden de un modo diferente a ciertos cuestionamientos, según Guba y Lincoln (1990) son tres las preguntas (Ver tabla 2) a realizar a cada uno de estos: ¿Cuál es la forma y la naturaleza de la realidad y, por lo tanto, ¿qué es lo que podemos conocer de ella? Esta es la pregunta ontológica; ¿Cuál es la naturaleza de la relación entre quien conoce o busca conocer y lo que puede ser conocido? Esta es la pregunta epistemológica y ¿Cómo puede el investigador (el que busca conocer) arreglárselas para averiguar si lo que él o ella cree puede ser conocido? Esta es la pregunta metodológica. La tabla 2 sintetiza así:

\footnotetext{
${ }^{4}$ Existe un salto o evolución de un positivismo a un post-positivismo que intenta, de algún modo, ser más flexible cuando trata o intenta afirmar ciertas verdades sobre la realidad, principalmente cuando estas afirmaciones son sobre lo social y humano, pues la realidad en esta dimensión es escurridiza y no se puede captar del todo. "La diferencia con el positivismo radica en que en el post-positivismo la realidad existe, pero no puede ser completamente aprehendida. Lo real se lo entiende desde las leyes exactas, únicamente puede ser entendida de forma incompleta. Una de las razones para no poder lograr una comprensión total y absoluta de la realidad se basa en la imperfección de los mecanismos intelectuales y perceptivos del ser humano, lo que lo limita para poder dominar todas las variables que pueden estar presentes en un fenómeno" (Ramos, 2015, p. 11).
} 
Tabla 2: Respuesta a las preguntas ontológica y epistemológica según cada paradigma.

\section{Post-positivista}

\section{Pregunta}

ontológica

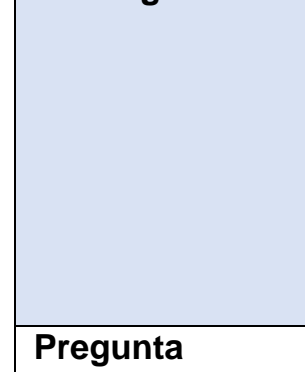

epistemológica

Dualista/objetivista modificada. Se abandona

el dualismo, en gran parte por ser imposible de sostener, pero la objetividad permanece como un "ideal regulador"; se hace un énfasis especial en "guardianes" externos de la objetividad, como las tradiciones críticas ( $¿$ "caben" los conocimientos dentro del conocimiento preexistente?) y la comunidad crítica (los editores, árbitros y colegas profesionales). Los hallazgos repetidos son probablemente reales (pero siempre sujetos a ser probados como falsos).

\section{Pregunta} Experimental/manipuladora modificada. Se metodológica hace un énfasis en la "pluralidad crítica" (una versión renovada de la triangulación) como forma de probar la falsedad (más que verificar) de las hipótesis.

\section{Interpretativo}

\section{Sociocrítico}

Relativista. Las realidades son
comprensibles en la forma de construcciones mentales múltiples e intangibles, basadas social y experiencialmente, de naturaleza local y específica (aunque con frecuencia hay elementos compartidos entre miembros de una comunidad e incluso entre distintas culturas), y su forma y contenido dependen de sujetos o grupos que sostienen esas construcciones.

Transaccional y subjetivista. Se supone que el investigador y el objeto de investigación están vinculados interactivamente de tal forma que los "hallazgos" son literalmente creados al avanzar la investigación. Desaparece la distinción. convencional entre ontología y epistemología, como sucede en el caso de la teoría crítica.

Hermenéutica y dialéctica. La naturaleza variable y personal (intramental) de las construcciones sociales sugiere que las construcciones individuales pueden ser producidas y refinadas sólo mediante la interacción entre el investigador y quienes responden. Estas construcciones variadas se interpretan utilizando técnicas hermenéuticas convencionales, y se comparan y contrastan mediante un intercambio dialéctico.
Realismo histórico. Se supone que es comprensible una realidad que anteriormente era plástica, pero a la que, a lo largo del tiempo, le han dado forma un cúmulo de factores sociales, políticos, culturales, económicos, étnicos y de género, para después quedar cristalizados (materializados) en una serie de estructuras que ahora se consideran (inapropiadamente) reales, es decir, naturales e inmutables.

Transaccional y subjetivista. Se supone que el investigador y el objeto investigado están vinculados interactivamente, y que los valores del investigador (y de los "otros" colocados) inevitablemente influencian la investigación. Por lo tanto los hallazgos están mediados por valores.

Dialógica y dialéctica. La naturaleza transaccional de la investigación requiere de un diálogo entre el investigador y lo investigado; ese diálogo debe ser de una naturaleza dialéctica para transformar la ignorancia y los conceptos erróneos (aceptando como inmutables a estructuras históricamente mediadas) en una conciencia más informada (al ver cómo se pueden cambiar las estructuras y entendiendo las acciones necesarias para efectuar el cambio). 
De la pregunta ontológica, el post-positivismo mantiene una posición de la realidad desde lo empírico y objetivo, como algo dado y que a su vez mantiene una relación íntima con un mundo visto como natural y que en ese espacio hondo yacen leyes que determinan el cauce de todo, incluyendo en mayor o menor medida lo social y humano. El paradigma interpretativo sostiene una subjetividad e incluso la enaltece, donde la realidad son construcciones mentales y la experiencia y los saberes aportan en dichas construcciones, en cuyo caso el relativismo acontece como una provocación epistemológica. Por último, el sociocrítico apunta a una realidad objetiva expresada como falsa conciencia, lo que, por contraste, quiere decir que hay en algún lugar una consciencia verdadera y que el investigador debe hallarla en relación dialógica con los sujetos con los que trabaja.

La educación, ciertamente, mantiene elementos empíricos y objetivos, de ahí que un paradigma post-positivista tenga cabida y sirva de referente para aludir a una dimensión de esta realidad; no obstante, su naturaleza, dinámica y desarrollo se destacan por mantener una íntima relación con las interacciones humanas, con las vicisitudes sociales, las interpretaciones y consecuentemente con los sentidos y significados, con las mentes que intentan darle sentido y significado. Los objetos de estudio, su construcción y reconstrucción, están sometidos a tal o cual ontología, el investigador tiene la obligación de establecer, con sus argumentos, las estructuras internas que les dan cuerpo y organizan.

En cuanto a la pregunta epistemológica, el post-positivismo contempla e incorpora un principio de objetividad, el cual como ideal se alcanza a partir de las constantes revisiones y vigilancias que deben estar firmes en el quehacer investigativo, con más o menos timidez, incorpora una racionalidad crítica con el fin de impulsar nuevos esquemas heurísticos a la investigación. El interpretativo pone a la comprensión, en un sentido profundamente hermenéutico y experiencial, como la clave del posicionamiento del investigador, objeto de estudio e investigador se fusionan en una dinámica de encuentro y desencuentro, de implicación, de modo que se logre comprender y describir el fenómeno estudiado. El sociocrítico de igual forma reposa en una visión subjetivista, donde el investigador se compromete con el objeto, solo que con implicaciones y fines éticos y morales, de transformación y mejora del mundo social. De los últimos dos paradigmas, parece haber mayor coincidencia, sobre todo al destacar el aspecto subjetivo, la diferencia es sustancial, la primera se vertebra en una filosofía hermenéutica, la segunda en una dialéctica. 
Tanto la razón ontológica como la epistemológica se entretejen en la construcción del objeto de estudio, donde la reconstrucción es aceptada con mayor entusiasmo en los paradigmas interpretativo y sociocrítico, pues hay un espíritu de reconocimiento de lo social y humano como algo indefinido, indeterminado e irreductible, pues el cambio y la transformación, ya sea en lo subjetivo y/o social, son una constante.

En cualquiera de los casos, la ontología y la epistemología de los paradigmas arriba mencionados marcan bases para el entendimiento de la naturaleza de la realidad y la manera en la que desde esa plataforma se puede construir conocimiento; sin embargo, los aportes que han abonado al campo no dejan de ser un referente que ayude a encontrar principios de acción en la construcción y reconstrucción del objeto de estudio.

\section{Reflexiones finales}

Domínguez (2007) recupera los aportes de Ángel Espinoza y Montes (1997), quien afirma que ciertamente hay un proceso de construcción reconstrucción del objeto de estudio, precisa que es una actividad que se realiza desde una sociología del conocimiento, diferenciada, e incluso distanciada de una visión de la filosofía de la ciencia, la cual es derivada y parte de una perspectiva positivista. La visión del autor asienta una reflexión epistémica-social, pues es en esas relaciones entre conocimiento y el proceso mismo de construcción de la investigación donde se encuentran los hitos que testimonian la construcción del objeto de estudio.

Rojas Soriano (2012, p.126), en una versión materialista dialéctica, establece que la relación que tenemos con el objeto de estudio contempla trece elementos, estos son:

1. Las características de este.

2. La concepción de la realidad que tengan tanto los directivos de los proyectos como los investigadores.

3. Las políticas y lineamientos de trabajo que marque la institución, esto sobre todo en los sectores público y privado.

4. El nivel de análisis y los objetivos de la investigación.

5. La disponibilidad de teorías sobre el tema y el alcance de estas.

6. La existencia de investigaciones empíricas y de experiencias relacionadas sobre el tema. 
7. El tipo de preguntas que se formulen y las hipótesis que se planteen, que dependen en gran medida de lo anterior.

8. La elección de los indicadores para orientar la búsqueda de la información empírica para comprobar las hipótesis.

9. El tipo de técnicas y las características de los instrumentos de recolección de datos que se utilicen.

10. El tiempo disponible para efectuar la investigación.

11. Los recursos financieros y materiales, así como el personal disponible y las características profesionales de este.

12. El tipo de preparación, experiencias, intereses y expectativas del investigador, que van a influir en:

13. La forma de interpretar y manejar todos los elementos anteriores.

Por su parte, Sánchez Puentes (1993) le otorga una importancia mayúscula y sustantiva al proceso de problematización como un camino sinuoso, el cual se alza como una actividad que incorpora de manera compleja métodos, técnicas, teoría, empírea y otros elementos que, entretejidos paulatinamente por el investigador, dan cuentas del problema y de lo que se pretende investigar.

Los objetos de estudio se constituyen como un referente de la investigación y en su momento más elaborado destaca una relación de orden epistemológico que tendrá implicaciones, relaciones dinámicas y precisas con los objetivos de conocimiento de la investigación, para Torres y Jiménez esta relación se expresa del siguiente modo:

Los objetivos de un proyecto de investigación expresan aquellos aspectos, relaciones, lógicas, dinámicas, efectos del problema, que pretenden ser esclarecidos con la investigación. En general, los objetivos de la investigación materializan la intención temática del investigador y corresponden a los aspectos por investigar provenientes del modelo de análisis, como las preguntas que quiere resolver el estudio. (2004, p.19)

En la actualidad, los Estados del Arte para Gómez, Galeano y Jaramillo (2015) su elaboración se han constituido como una etapa determinante y neurálgica en toda investigación, como una actividad que recolecta las distintas visiones, formas de entender, de abordar y trabajar con problemáticas y objetos de estudio similares, de manera que esto 
permite, entre otras cosas, una reflexión sobre la forma en la que se construye el objeto de estudio, y en dado caso de ser necesario, una reconstrucción del mismo de modo que se logre uno más adecuado a los intereses y expectativas.

En cualquiera de los casos, ya sea de los paradigmas interpretativo y sociocrítico fundamentalmente, construir el objeto de estudio es una actividad que atraviesa diversas etapas que son el resultado de una relación dinámica y compleja del investigador con su universo de trabajo, actividad que incorpora tanto elementos que van desde recursos de corte cientificista hasta datos empíricos que sean verosímiles para los fines de la investigación, también el carácter subjetivo adquiere una importancia distintiva, al ser el mundo de lo social y humano un espacio de cambio y contradicción constante.

Algunos marcos epistemológicos alternativos, emergentes en la actualidad, ofrecen posibilidades distintas a la discusión de la construcción del objeto de estudio; el primero, constituido por el anarquismo epistemológico de Paul Feyerabend (1993 y 1996), propone una versión de la construcción del conocimiento bajo la premisa de "todo se vale"; por otra parte, Umberto Eco (1999) incorpora a la discusión la difícil tarea de entender la semiótica, pues nos referimos a un algo y pensamos ¿qué es ese algo y qué nos convoca a pensar sobre ese algo? así:

¿Por qué la semiótica debería ocuparse de ese algo? Porque uno de sus problemas es (también, y sin duda) decir si y cómo usamos signos para referirnos a algo, aspecto del cual se ha escrito mucho. Pero no creo que la semiótica pueda evitar otro problema: ¿qué es ese algo que nos induce a producir signos? (1999, p.29)

Boaventura Do Sousa Santos (2009) realiza una importante y significativa pretensión de reivindicación del saber local, originario y de cotextos latinoamiericanos fundamentalmente, referido al saber segregado históricamente.

Como aporte a la discusión, para poder entender la construcción y reconstrucción del objeto de estudio, antes, es necesario concebir la dimensión ontológica de los paradigmas: cuestionar la realidad y preguntarnos qué es y qué no es real, qué hace que algo lo sea, qué características debe cubrir y cómo se puede eso sostener en una investigación como objeto de estudio y que a su vez está en constante transformación; con la primicia sustancial de que lo social y humano está en constante metamorfosis. 
En el mismo tenor, y para poder contribuir al entendimiento de la construcción y reconstrucción del objeto de estudio, sobre todo en espacios educativos, hay que destacar que las escuelas son sitios de encuentro y reencuentro con el otro, lugares donde la subjetividad se encuentra con otra, y propicia que este sea un lugar de intercambio de ideas y que como consecuencia se logre una transformación de la persona, ya sea de estudiantes, planta académica o cualquiera de los implicados en estos lugares.

La experiencia vivida, como un elemento que destaca las condiciones a priori del pensamiento sobre el objeto, como ese espacio intuitivo del ser humano en el mundo en su condición vivencial, ofrece posibilidades para comprender aspectos de la educación que en su estudio, indagación o investigación suelen ser contingentes y por lo tanto de difícil objetivación, que se hacen irreductibles. En esta visión de lo educativo, valdría la pena resaltar los aportes de la fenomenología al campo científico desde un paradigma interpretativo, aporte que intenta destacar las posibilidades descriptivas del mundo de la vida, mediante el arte del lenguaje, esto aunado con la hermenéutica posibilitan la comprensión por la develación de sentidos y significados

[...] la fenomenología es el estudio del mundo de la vida, es decir, del mundo tal como lo experimentamos inmediatamente de un modo prereflexivo, y no tal como lo conceptualizamos o categorizamos, ni como reflexionamos sobre él. [...] La fenomenología pregunta: «¿Cómo es tal o cual tipo de experiencia?». (Van Manen, 2003, p. 27)

Las escuelas, entendidas como espacios de experiencias, subjetividades, saberes, cultura e historia, son sitios donde las contingencias, vicisitudes, contradicciones y cambios son una constante. En esta panóptica de la educación se comprende la realidad como algo incierto e incluso abierto a las interpretaciones, a los puntos de vista y a afirmaciones sostenidas desde la fuerza de las argumentaciones. La faena educativa se compone sustancialmente de subjetividad, de vida y pasión, investigar la educación, construir un objeto de estudio y sus reconstrucciones exige un ánimo del investigador que permita la apertura de sus sentidos, pero sobre todo, de su sentir ante el fenómeno en cuestión, es aceptar que no hay nada dado ni acabado en lo social y humano, por el contrario, la temporalidad, el cambio y la contradicción son, entre otros principios, el cuadro epistemológico que determina su quehacer investigativo. "Lo serio es valorar el corazón por 
encima de la lógica y finalmente, aceptar que el mundo del ser humano no es otro que el de cotidianidad, desnudo de referencias a montajes tecnocientíficos o metafísicos" (Fullat, 2002, p. 293).

La ciencia, tanto en la enseñanza y aprendizaje de los conocimientos como en el desarrollo mismo de la actividad investigativa, atraviesa irremediablemente por una dimensión profundamente humana, por una preocupación de vida, una curiosidad, una duda, por un espíritu de búsqueda de la verdad que bien pueden ser recuperada por un profesor, José Manuel Esteve (1998), lo expresa del siguiente modo: "Todas las ciencias tienen en su origen a un hombre o una mujer preocupados por desentrañar la estructura de la realidad. Alguien, alguna vez, elaboró los conocimientos del tema que explicas, como respuesta a una preocupación vital" (p. 46).

Construir y reconstruir un objeto de estudio incorpora en su actividad la curiosidad y las ganas de entender algo, un fenómeno de la realidad. Edmund Halley, por ejemplo, cuando era niño vio en el cielo un astro que lo asombró -en un contexto donde la superstición predominaba y era la base de las explicaciones e incluso avistamientos de este tipo se relacionaban significativamente con catástrofes y muerte- creció y pudo estudiar y dotarse de los conocimientos necesarios, investigó sobre aquel objeto en el cielo y descubrió que los testimonios de otras civilizaciones en distintos tiempos en realidad eran sobre el mismo objeto, y según sus cálculos este cometa pasaba cerca de nuestro planeta cada setenta y cinco años, e incluso predijo el día y la hora que sucedería de nuevo, pocos le creyeron, luego de su muerte algunos de sus seguidores se reunieron en la fecha señalada y para la sorpresa de propios y extraños dicho cometa sí pasó, en su honor se llama Cometa Halley. Así, el astrónomo inglés nos enseña, entre otras cosas, que un objeto de estudio puede tener otras explicaciones y cada una nos acerca más a la verdad.

En la educación cada fenómeno, cada suceso que se vive en las escuelas, puede ser el objeto de estudio de alguien y su construcción y reconstrucción será una actividad que se sustenta por un paradigma y su respectiva epistemología y ontología, pero también es una acción profundamente humana, desde la curiosidad, el deseo y el ánimo de querer indagar sobre eso, una inquietud vital, propia y personal de quien hace la actividad investigativa. 


\section{Referencias}

Chalmers, Alan Francis. (1992). ¿Qué es esa cosa llamada Ciencia? Una valoración de la naturaleza y el estatuto de la ciencia y sus métodos. México: Siglo XXI.

de Garay, Adrián. (2004). Los actores desconocidos. Una aproximación al conocimiento de los estudiantes. México: Asociación Nacional de Universidades e Instituciones de Educación Superior.

de Garay, Adrian. (2008). Los jóvenes universitarios mexicanos: ¿son todos iguales? En M. Suárez y J. Pérez, Jóvenes universitarios en latinoamérica, hoy (pp. 205-222). México: Miguel Ángel Porrúa.

Díaz Barriga, Ángel. (1997). La explicación científica. Una polémica desde la teoría del conocimiento. En Carlos Ángel, Hoyos, Epistemología y objeto pedagógico ¿Es la pedagogía una ciencia? (pp. 135-149). México: Plaza y Valdéz.

Do Sousa Santos, Boaventura. (2009). Una epistemología del sur. La reinvención del conocimiento y la emancipación social. México: Siglo XXI.

Domínguez, Silvia. (2007). El objeto de estudio en la investigación. Diversas aproximaciones. Revista de Educación y Desarrollo, (7), 41-50. Recuperado de http://www.cucs.udg.mx/revistas/edu desarrollo/anteriores/7/007 Dominguez.pdf

Ducoing, Patricia. (2016). La investigación en educación: epistemologías y metodologías. México: Asociación Francófona Internacional de Investigación en Ciencias de la Educación. Sección mexicana (AFIRSE); Plaza y Valdés S.A. de C.V.

Eco, Umberto. (1999). Kant y el ornitorrinco: Madrid, España: Lumen.

Espinoza y Montes, Ángel. (1997). Conocimiento e investigación: necsidades epistémicas del proceso de enseñanza aprendizaje. En Carlos Ángel Hoyos, Epistemología y objeto pedagógico ¿Es la pedagogía una ciencia? México: Plaza y Valdés.

Esteve, José Manuel. (1998). La aventura de ser profesor. En Cuadernos de pedagogía, (266). Barcelona, España. Recuperado de https://www.ugr.es/ fjjirios/pce/media/6AventuraSerProfesor.Esteve.pdf

Feyerabend, Paul. (1993). Tratado contra el método. Esquema de una teoría anarquista del conocimiento. México: REI México.

Feyerabend, Paul. (1996). Adios a la razón. Madrid, España: Editorial Tecnos, S.A.

Fullat, Octavi. (2002). Pedagogía existencialista y posmoderna. España: Síntesis.

Gómez, Maricely., Galeano, Catalina. y Jaramillo, Dumar. (2015). El estado del arte: una metodología de investigación. Revista Colombiana de Ciencias Sociales, 6(2), 423-442. Recuperado de https://dialnet.unirioja.es/descarga/articulo/5212100.pdf 
González, María. (2011). Las trayectorias de los estudiantes universitarios: un modelo integral. Montreal, Canadá: Université de Montréal. Département d'administration et fondements de l'éducation.

Guba, Egon. y Lincoln, Yvonna. (1990). Paradigmas en competencia en la investigación cualitativa. En N. Denzin e Y. Lincoln, Handbook of Qualitative Research. California, United States: Thousand Oaks.

Habermas, Jürgen. (1984). Ciencia y técnica como ideología. Madrid, España: Tecnos.

Kuhn, Thomas. (2000). Las estructuras de las revoluciones científicas. México: FCE.

Larrosa, Jorge. (2003). La experiencia de la lectura: estudios sobre literatura y formación. México: FCE.

Mardones, José María. y Ursúa, Nicanor. (1995). Filosofía de las ciencias sociales y humanas. Materiales para una fundamentación científica. México: Fontamara S.A.

Ramos, Carlos Alberto. (2015). Los paradigmas de la investigación científica. UNIFE, 1(25), 9-25. Recuperado de https://www.researchgate.net/profile/Carlos RamosGalarza/publication/282731622 LOS PARADIGMAS DE LA INVESTIGACION CIEN TIFICA Scientific research paradigms/links/561a519d08ae044edbb208fe/LOSPARADIGMAS-DE-LA-INVESTIGACION-CIENTIFICA-Scientific-research-pa

Rojas Soriano, Raúl. (2012). Métodos para la investigación social. Una proposición dialéctica. México: Plaza y Valdés Editores. Recuperado de https://raulrojassoriano.com/cuallitlanezi/wpcontent/themes/raulrojassoriano/assets/libros/metodos-investigacion-social-rojassoriano.pdf

Sánchez Puentes, Ricardo. (1993). Didáctica de la problematización en el campo científico de la educación. Perfiles Educativos, (61), 64-78. Recuperado de http://www.iisue.unam.mx/perfiles/descargas/pdf/1993-61-64-78

Sartre, Jean-Paul. (2012). La nausea. México: Losada.

Schutz, Alfred. (2015). El problema de la realidad social. México: Amorrortu.

Torres, Alfonso., y Jiménez, Absalón. (2004). La construcción del objeto y los referentes teóricos en la investigación social. En Alfonso Torres y Absalón Jiménez, La práctica investigativa en ciencias sociales (pp. 13-26). Bogota: UPN, Universidad Pedagógica Nacional, Consejo Latinoamericano de Ciencias Sociales (CLACSO). Recuperado de http://bibliotecavirtual.clacso.org.ar/ar/libros/colombia/dcsupn/practica.pdf

Van Manen, Max. (2003). Investigación educativa y experiencia vivida: Ciencia humana para una pedagogía de la acción y la sensibilidad. Barcelona: Idea Books, S.A.

Tinto, Vincent. (1987). Leaving College. Rethinking the causes and cures of student. Chicago, United States: The University of Chicago Press. 
Revista indizada en

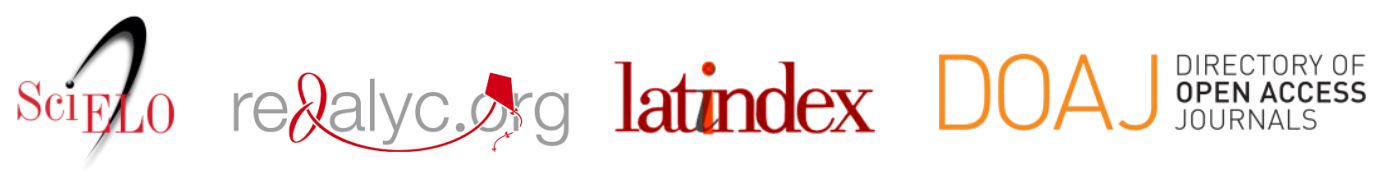

Distribuida en las bases de datos:

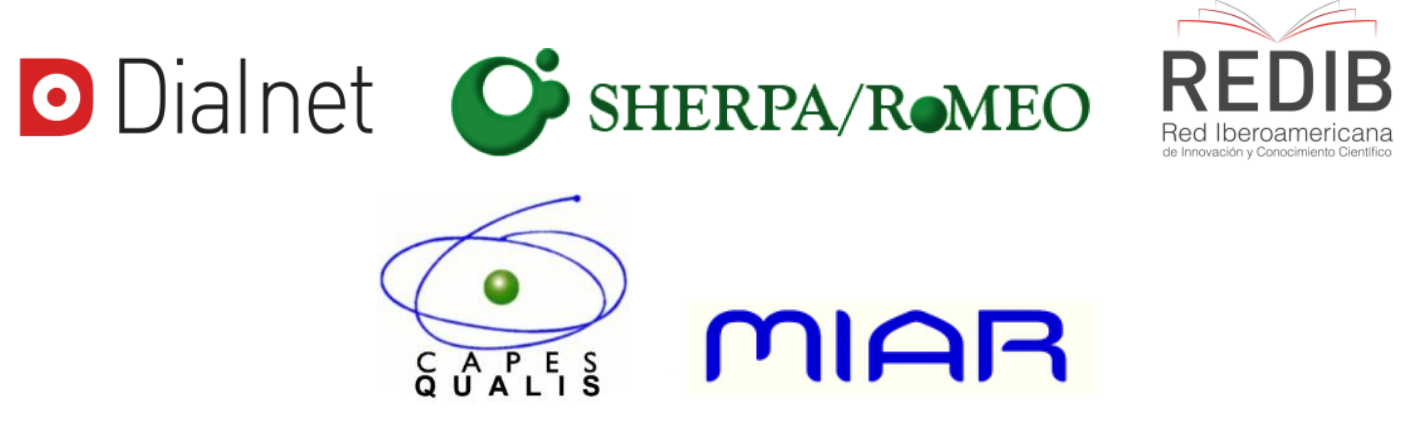

